\title{
SPECTRAL-SPATIAL JOINT SPARSITY UNMIXING OF HYPERSPECTRAL DATA USING OVERCOMPLETE DICTIONARIES
}

\author{
J. Bieniarz, E. Aguilera, X. X. Zhu, R. Müller, U. Heiden, P. Reinartz \\ German Aerospace Center (DLR), Oberpfaffenhofen, 82234 Wessling, Germany.
}

\begin{abstract}
Sparse spectral unmixing can be modeled as a linear combination of endmembers contained in an overcomplete dictionary weighted by the corresponding sparse abundance vector. This method exploits the fact that there is only a small number of endmembers inside a pixel compared to the overcomplete endmember spectral dictionary. Since the information contained in hyperspectral pixels is often spatially correlated, in this work we propose to jointly estimate the sparse abundance vectors of neighboring hyperspectral pixels within a local window exploiting joint sparsity with common and noncommon endmembers. To demonstrate the efficiency of our framework, we perform experiments using both simulated and real hyperspectral data.
\end{abstract}

Index Terms - Spectral unmixing, joint sparsity, overcomplete spectral dictionary.

\section{INTRODUCTION}

A common approach to model pixels of hyperspectral images (HSI) exploits a linear mixing of spectral signatures referred to as endmembers. These signatures correspond to the spectra of pure materials, which can be obtained from field/laboratory spectrometer measurements [1] or determined from the data by exploiting pixels known to contain a single material. The basic linear mixing model will be assumed to be given by

$$
y_{j}=A x_{j}+\varepsilon_{j}
$$

where $y_{j} \in \mathbb{R}_{\geq 0}^{m}$ denotes the reflectance of the $j$ th hyperspectal pixel with $m$ spectral channels; $A \in \mathbb{R}_{\geq 0}^{m \times n}$ is an overcomplete spectral dictionary [2] of $n$ endmembers (i.e., $n$ is inevitably greater than $m) ; x_{j} \in \mathbb{R}_{\geq 0}^{n}$ represents the abundance vector to be estimated; and $\varepsilon_{j} \in \mathbb{R}^{m}$ is a perturbation term that captures any modeling errors and noise contributions [1]. Thus, given $y_{j}$ and $A$, the goal is to recover the unknown vector $x_{j}$.

Traditional inversion techniques include the method of least squares (LS), nonnegative least squares (NNLS) [1], and fully-constrained least squares (FCLS) [3]. While NNLS

This work is associated with the project SparsEO funded by Munich Aerospace e.V. Fakultät für Luft- und Raumfahrt. ensures the LS criterion is restricted to nonnegative abundances, FCLS additionally enforces that the elements of the abundance vector sum to one. Although often employed, these techniques are limited to overdetermined mixing models and thus require a preselection of endmembers based on a priori knowledge [1]. In contrast, recent sparsity-based unmixing algorithms have been shown to be able to deal with the underdetermined case [2, 4, 5]. In essence, these techniques exploit the fact that-assuming a suitable spatial resolution—only few endmembers contribute to $y_{j}$. In other words, high-resolution imaging renders the vector $x_{j}$ sparse. Accordingly, recovery of abundances is usually achieved by solving optimization problems such as basis pursuit denoising (BPDN) [6] or the least absolute shrinkage and selection operator (LASSO) [7].

Spatial contextual homogeneity is exploited by applying an alternative joint sparsity model (JSM) originally introduced in [8]. In this setting, every vector $x_{j}$ will be recovered together with a small number of neighboring abundance vectors selected by means of a window centered on the $j$ th spatial position. The basic inputs to the algorithm are then multiple neighboring pixels $y_{j}$ (hereinafter referred to as looks) as well as a dictionary $A$. Thus, the goal will be to take advantage of the fact that the ensemble of unknown vectors exhibit both common endmembers and abundances. As will be shown, this approach also allows for capturing their noncommon endmembers. Finally, the applicability of JSM will be validated by comparing it with BPDN, NNLS using both real and simulated data.

\section{MULTI-LOOK MODEL FOR SPARSE SPECTRAL UNMIXING}

Given the linear model defined by equation (1) and under the assumption of a small number of active endmembers (i.e., sparsity), the nonnegative BPDN algorithm [2, 4,5] estimates a single abundance vector $x_{j}$ (i.e., a single look) by solving the following $L_{1}$-norm optimization problem:

$$
\min _{\widetilde{x}_{j}} \frac{1}{2}\left\|A \widetilde{x}_{j}-y_{j}\right\|_{2}^{2}+\lambda\left\|\widetilde{x}_{j}\right\|_{1} \text { s.t. } \widetilde{x}_{j} \geq 0
$$

Since the $L_{1}$-norm serves as a proxy for sparsity, the penalty parameter $\lambda$ can be used to trade sparsity of the solution for 
data mismatch. Also, in order to ensure physical validity, the abundance vector is constrained to be nonnegative. Furthermore, note that the performance of (2) can be shown to depend on the conditioning of $A$, the sparsity level, and the perturbation level (see, for example, [1, 5, 9] and the references therein).

As it follows from (2), BPDN operates on a pixel-by-pixel basis and is thus unaware of the spatial ergodicity commonly exhibited by most pure materials. In effect, endmember abundance is expected to present a slow variation along the two spatial dimensions. Thus, given a $L$-element window centered on the $j$ th position, the $i$ th pixel $y_{i}$ (with $1 \leq i \leq L$ ) can be expressed as

$$
y_{i}=A x_{i}=A x_{j}^{\mathrm{c}}+A x_{i}^{\mathrm{nc}}
$$

where $x_{j}^{\mathrm{c}} \in \mathbb{R}_{\geq 0}^{n}$ and $x_{i}^{\mathrm{nc}} \in \mathbb{R}^{n}$ denote, respectively, the common and noncommon abundances. By letting

$$
Y_{j}=\left[\begin{array}{c}
y_{1} \\
y_{2} \\
\vdots \\
y_{L}
\end{array}\right] \quad X_{j}=\left[\begin{array}{c}
x_{j}^{\mathrm{c}} \\
x_{1}^{\mathrm{nc}} \\
\vdots \\
x_{L}^{\mathrm{nc}}
\end{array}\right]
$$

and

$$
A_{\text {JSM }}=\left[\begin{array}{ccccc}
A & A & 0 & \cdots & 0 \\
A & 0 & A & \cdots & 0 \\
\vdots & \vdots & \vdots & \ddots & \vdots \\
A & 0 & 0 & \cdots & A
\end{array}\right]
$$

the resulting multi-look linear model can be written as

$$
Y_{j}=A_{\mathrm{JSM}} X_{j}+Z_{j}
$$

where $Z_{j} \in \mathbb{R}^{L m}$ accounts for the corresponding $L$ perturbations and $A_{\text {JSM }} \in \mathbb{R}_{\geq 0}^{L m \times(L+1) n}$ is a JSM dictionary [8]. Accordingly, under the hypothesis of the existence of common components, $X_{j}$ provides a sparse representation that exploits the redundancy exhibited by the $L$ sparse vectors $x_{i}$. As a result, the multi-look JSM reconstruction (MLJSR) can be accomplished by means of the previously introduced nonnegative version of BPDN:

$$
\min _{\widetilde{X}_{j}} \frac{1}{2}\left\|A_{\mathrm{JSM}} \widetilde{X}_{j}-Y_{j}\right\|_{2}^{2}+\lambda\left\|\widetilde{X}_{j}\right\|_{1} \text { s. t. } \widetilde{X}_{j} \geq 0
$$

where, again, $\lambda$ is used to trade sparsity for data mismatch.

By way of illustration, consider the case of $L$ abundance vectors $x_{i}$ containing a single common material. Next, assume their abundances are the same except for one vector (hereinafter indicated by $x_{0}$ ) which presents a lower abundance. It then follows that, by allowing negative noncommon components, the minimum achievable support of $X_{j}$ reduces to 2 . On the contrary, by enforcing nonnegativity of $X_{j}$, the minimum achievable support equals $L$. Nonetheless, if $x_{0}$ is high enough, either case will result in one dominant common abundance and hence the residual components can be assumed to be captured by the perturbation term $Z_{j}$. In light of the previous considerations, this paper will provide a numerical evaluation of MLJSR based on (7).

\section{EXPERIMENTAL RESULTS}

We have tested our algorithm using simulated and real hyperspectral data. For the simulation experiment, we used 90 manmade materials, vegetation, and soil spectra selected from the USGS spectral dictionary [10], merged with endmembers from a DLR roof-material dictionary containing 39 endmembers into one spectral dictionary $A$ with finally $m=129$ endmembers. After discarding noisy and water-absorption bands, each spectrum in the dictionary exhibits $n=96$ bands.

\subsection{Simulated Scenario}

In order to assess the performance of the MLJSR algorithm with hyperspectral data using the dictionary $A$, we simulated 10000 joint $Y_{L}$ pixel ensembles each with $L=4$ pixels. The pixels $y_{1}, \ldots, y_{4}$ form a mixture of $2,3,3$, and 4 endmembers, respectively, and share 2 common endmembers. The common and noncommon endmembers were randomly selected from the dictionary $A$ and the abundances for all pixels were also randomly chosen with the sum to one restriction. The abundance of each common endmember was at least $10 \%$. Additionally, the simulated pixels were contaminated with white noise to achieve a signal-to-noise ratio (SNR) of 25 , 30, 40, 50, and $60 \mathrm{~dB}$, respectively. The SNR, signal-toreconstruction error (SRE) are defined as in [9]. The errors are calculated for pixel $y_{4}$ in all simulated $Y_{L}$, as the most mixed pixels contain 2 common and 2 noncommon endmembers. Fig. 2 presents results for the simulated pixels experiment using both MLJSR and BPDN method. The function of mean SRE for 10000 simulated pixels with different SNR is plotted. The proposed MLJSR outperforms NNLS as well as BPDN for all tested noise conditions.

Subsequently we performed unmixing with MLJSR and BPDN arranging the $\lambda$ parameters in the range from $e^{-15}$ to 1. For $\lambda<e^{-15}$ solution stabilizes for all tested scenarios so that the $L_{1}$ term of the minimization (2) does not have significant influence on the final result and becomes equivalent to NNLS, i.e., when $\lambda=0$. The upper bound of $\lambda=1$ indicates the direction towards unrealistic solutions. In Fig. 1 the SRE for MLJSR and BPDN at different noise levels is shown.

The MLJSR outperformed the BPDN for all test cases, i.e., the maximum SRE for MLJSR is always greater than the maximum SRE for BPDN. Additionally, we can observe that the best reconstruction in terms of SRE is dependent on $\lambda$ and the noise level. For smaller SNR stronger regularization is required. 


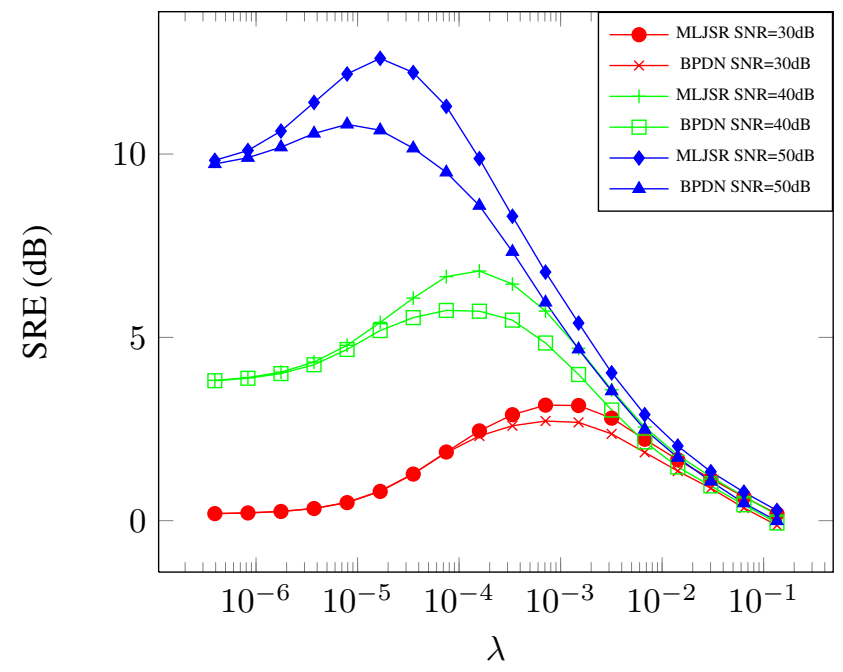

Fig. 1. Plot of the SRE values as a function of $\lambda$ regularization parameter for reconstruction of abundance from simulated pixels with SNR $=30,40,50 \mathrm{~dB}$ using BPDN and MLJSR methods.

\subsection{Real HyMap Image}

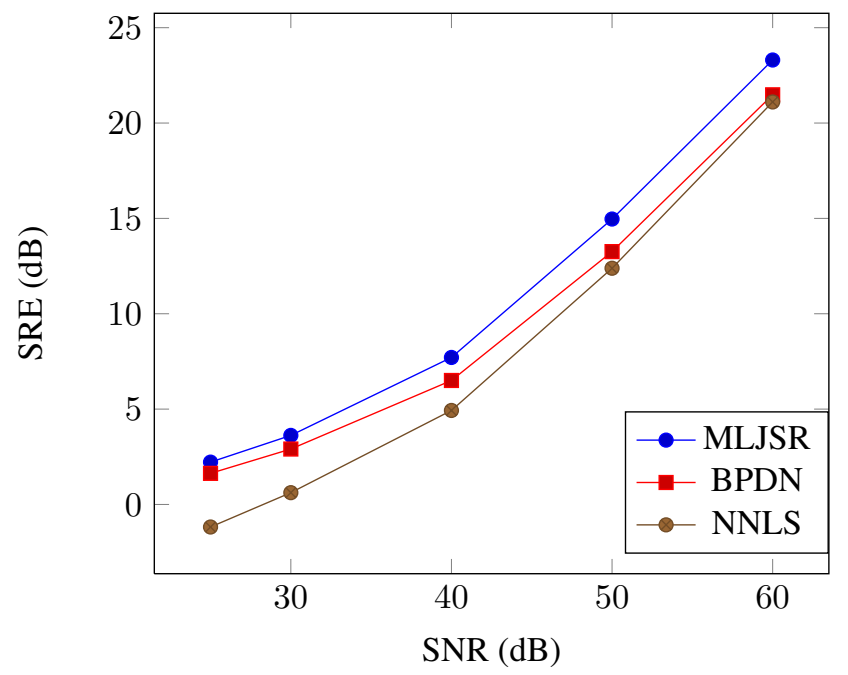

Fig. 2. Comparison of NNLS, BPDN, and MLJSR algorithms using (a) SRE and (b) nRMSE measures for signal with different SNR.

For the experiment with real data we used a hyperspectral scene acquired over Dresden, Germany, with the airborne sensor HyMap [11]. The scene with originally 126 spectral channels was reduced to 96 . The image has been atmospherically and geometrically corrected with a ground resolution of $4 \mathrm{~m}$. The window size for MLJSR was $L=9$ where the unmixed pixel $y_{i, j}$ was in the center of the window. By sliding the window over the image each pixel was processed, i.e., we used measurements of all 9 pixels to get a reliable unmixing only for the central pixel.

We have tested both MLJSR and BPDN with $10 \lambda$ parameters arranged in the range from $e^{-9}$ to $e$. In order to select the best reconstruction, we have overlapped all unmixing results on the high resolution image and empirically selected the $\lambda$ parameter which provided the best results according to our knowledge. The qualitative comparison of the unmixing results is presented in Fig. 3 These results were computed using NNLS (right column), both BPDN (middle column) and MLJSR (left column) with $\lambda=0.13$. The MLJSR algorithm in comparison to BPDN and NNLS results in smoother transitions from one material to another which indicates better recovery of mixed pixels with small abundances for selected materials. This benefits from the joint recovery of neighboring pixels.

\section{CONCLUSION}

In this paper we have proposed a multi-look joint sparse reconstruction for sparse spectral unmixing. The proposed model takes advantage of the neighboring information by means of joint approximation of abundances in a sliding window and especially a joint approximation of common endmembers. Compared to the standard sparse BPDN or NNLS method, experiments with simulated data demonstrate that the proposed method restores abundances for pixels sharing common endmembers more accurately in terms of the SRE measure. The qualitative analysis of the results on HyMAP image shows the MLJSR approach to be competitive with respect to BPDN and NNLS methods and visually show more consistent results. As future work, we propose to address further tests on real hyperspectral data including quantitative analysis. Additionally, the MLJSR can be extended to a more complex design, e.g. incorporating not only common endmembers in the whole window but also shared endmembers between singular pixels in the window.

\section{REFERENCES}

[1] J.M. Bioucas-Dias, A. Plaza, N. Dobigeon, M. Parente, Qian Du, P. Gader, and J. Chanussot, "Hyperspectral unmixing overview: Geometrical, statistical, and sparse regression-based approaches," Selected Topics in Applied Earth Observations and Remote Sensing, IEEE Journal of, vol. 5, no. 2, pp. 354 -379, Apr. 2012.

[2] M.-D. Iordache, J. M. Bioucas-Dias, and A. Plaza, "Sparse unmixing of hyperspectral data," Geoscience and Remote Sensing, IEEE Transactions on, vol. 49, no. 6, pp. 2014-2039, 2011.

[3] D.C. Heinz and Chein-I-Chang, "Fully constrained least squares linear spectral mixture analysis method for ma- 


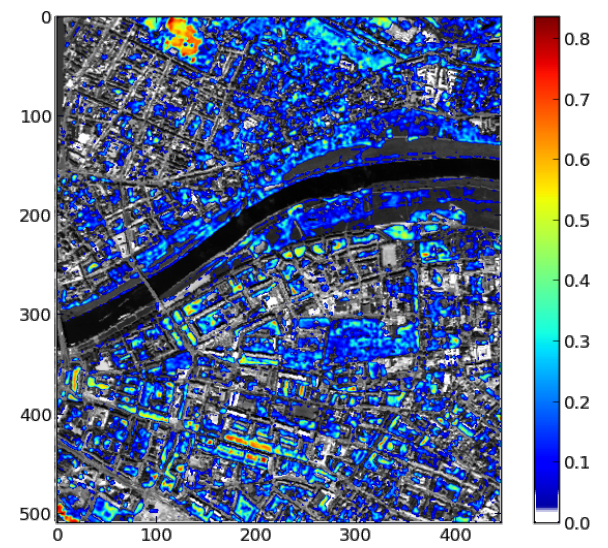

(a)

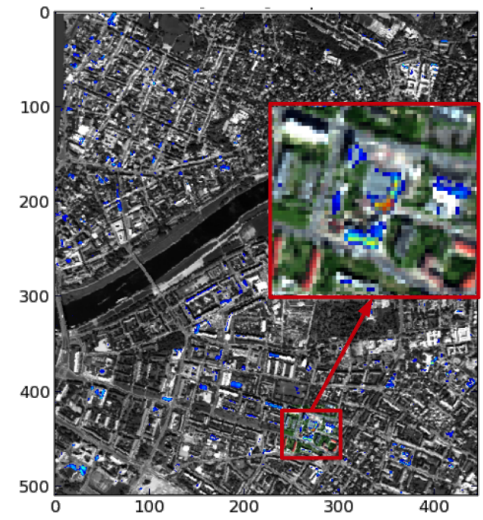

(d)

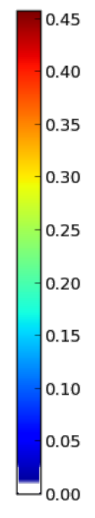

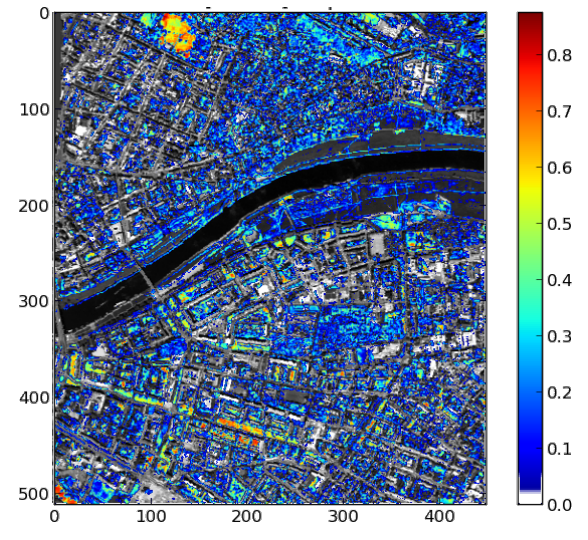

(b)

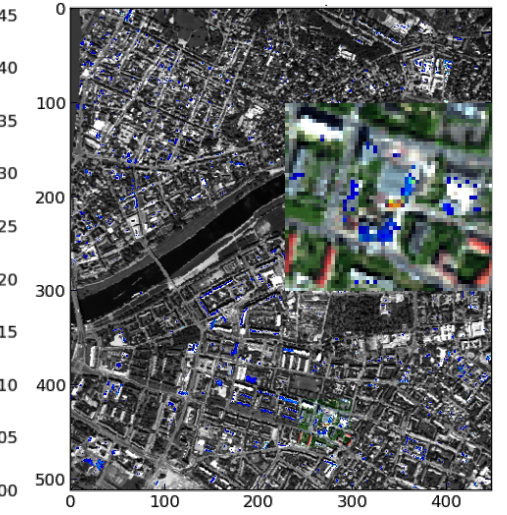

(e)

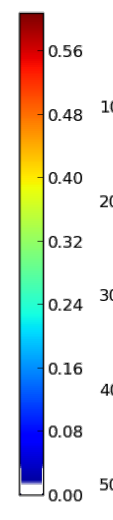

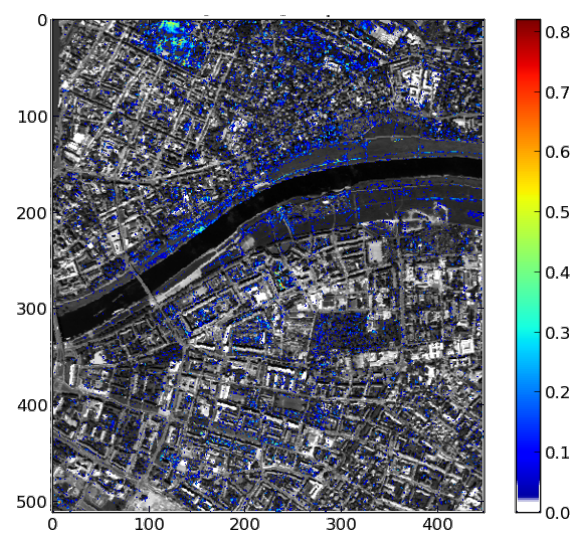

(c)

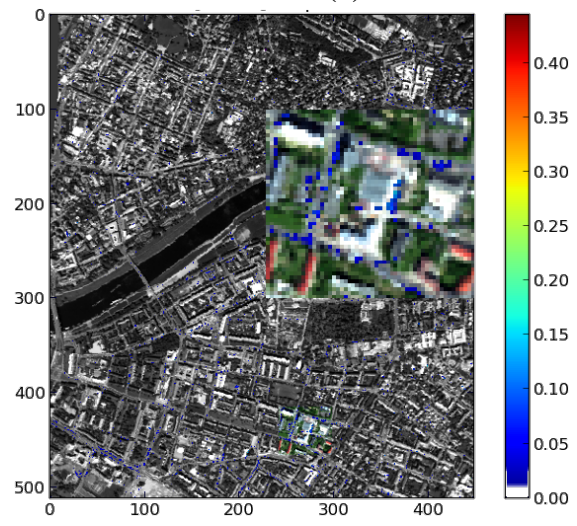

(f)

Fig. 3. Unmixing results for, respectively columns from left to right, MLJSR, LASSO and NNLS. First row of images presents abundance map for white roof shielding. The second row are the abundances for lawn grass.

terial quantification in hyperspectral imagery," Geoscience and Remote Sensing, IEEE Transactions on, vol. 39, no. 3, pp. $529-545$, Mar. 2001.

[4] M. Parente and A. Zymnis, "Statistical clustering and mineral spectral unmixing in aviris hyperspectral image of cuprite, nv," Tech. Rep. CS229, Stanford University, December 2005.

[5] J. Bieniarz, R. Müller, X. X. Zhu, and P. Reinartz, “On the use of overcomplete dictionaries for spectral unmixing," in 4th Workshop on Hyperspectral Image and Signal Processing. WHISPERS 2012, June 2012.

[6] Scott Shaobing Chen, David L. Donoho, Michael, and A. Saunders, "Atomic decomposition by basis pursuit," SIAM Journal on Scientific Computing, vol. 20, pp. 3361, 1998.

[7] Robert Tibshirani, "Regression shrinkage and selection via the lasso," Journal of the Royal Statistical Society, Series B, vol. 58, pp. 267-288, 1994.
[8] D. Baron, M. F. Duarte, S. Sarvotham, M. B. Wakin, and R. G. Baraniuk, "An information theoretic approach to distributed compressed sensing," in Allerton Conference on Communication, Control, and Computing, 2005.

[9] M.-D. Iordache, J.M. Bioucas-Dias, and A. Plaza, “Collaborative sparse regression for hyperspectral unmixing," Geoscience and Remote Sensing, IEEE Transactions on, vol. PP, no. 99, pp. 1-14, 2013.

[10] R.N. Clark, G.A. Swayze, R. Wise, E. Livo, T. Hoefen, R. R. Kok, and S.J. Sutley, "Usgs digital spectral library splib06a," Digital Data Series 231, 2007.

[11] T. Cocks, R. Jenssen, A. Stewart, I. Wilson, and T. Shields, "The hymap (tm) airborne hyperspectral sensor: the system, calibration and performance," in $1 s t$ EARSeL Workshop on Imaging Spectroscopy. EARSeL, 1998. 